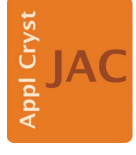

JOURNAL OF APPLIED CRYSTALLOGRAPHY

ISSN 1600-5767

Keywords: book reviews.

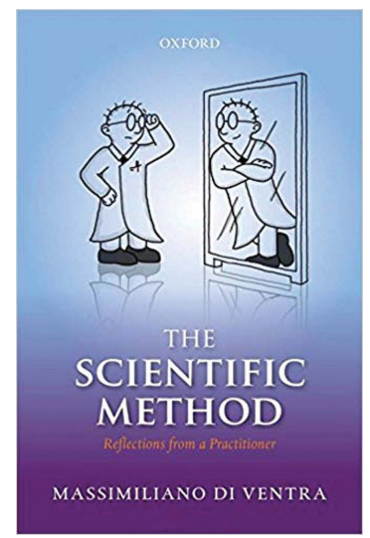

(C) 2018 International Union of Crystallography

\section{The Scientific Method: Reflections from a Practitioner. By Massimiliano di Ventra. Oxford University Press, 2018. Pp. 128. Price GBP 13.99. ISBN 9780198825623.}

John R. Helliwell*

Department of Chemistry, University of Manchester, Manchester M13 9PL, UK. *Correspondence e-mail: john.helliwell@manchester.ac.uk

The contents of this short book involve 18 chapters and 128 pages. The chapter headings are 1. Science Without Philosophy, 2. Material World and Objective Reality, 3. First Principles and Logic, 4. Natural Phenomena and the Primacy of Experiment, 5. Observation and Experimentation, 6. The Role of Human Faith in Science, 7. Approximate and Limited Description of Natural Phenomena, 8. Hypothesis, 9. Theory, 10. Competing Theories, 11. Can One Theory Be 'Derived' from Another?, 12. Verifying or Falsifying? And What?, 13. Don't Be a Masochist!, 14. 'Consensus' in Science? What Is That?, 15. Flow Chart of the Scientific Method, 16. The 'What' and 'Why' Questions, 17. 'Scientism': Abusing the Scientific Method, 18. Final Thoughts.

The back page of the book describes it as follows:

This book looks at how science investigates the natural world around us. It is an examination of the scientific method, the foundation of science, and basis on which our scientific knowledge is built on. Written in a clear, concise, and colloquial style, the book addresses all concepts pertaining to the scientific method. It includes discussions on objective reality, hypotheses and theory, and the fundamental and inalienable role of experimental evidence in scientific knowledge.

From the author's university web page I learnt that he is a theoretical physicist and has a strong publication record: for ease and simplicity I quote his h-index of 63 . The author concludes his Preface with 'My hope is that this book will rekindle a much needed interest in the centuries-old structure and foundations of this marvellous human enterprise we call Science.' Has the fire gone out? Let us find out....

Chapter 1 sets the scene by describing that science and philosophy are different. This concept is most neatly summed up on page 13 at the end of chapter 2: 'All scientific statements need to be logical, but not all logical statements need to be scientific.' Chapter 2 also sets out boldly and clearly that there is an objective reality: 'Denying the existence of an objective reality that is present irrespective of the observer is equivalent to denying the very existence of Science and its method.' This sentence is preceded by the author's statement that 'I have encountered people that would deny the existence of such an objective reality. Although this is often the subject of movies or some 'beliefs', I cannot truly fathom a scientist's claiming the same.' I would definitely agree. Chapter 3 is entitled First Principles and Logic and 'summarizes the core of what is called common sense'. It is made clear by di Ventra, for example, that 'For any proposition, either that proposition is true or its negation is true' and 'For an event to occur at a given time, there must be another event that caused it.' Then the chapter usefully makes clear that a scientific description 'needs to lead to testable predictions'. Chapter 4, Natural Phenomena and the Primacy of Experiment, opens with a cartoon sketch of the leaning tower of Pisa linking, I imagine, to Galileo's experiment of the late 16th century that two objects of different masses would both fall to the ground at the same time as a result of their experiencing the same acceleration due to gravity. That picture is somewhat undermined when on page 34 in chapter 5 the author outlines the situation where two objects of the same mass fall to the ground at the same time, excluding air resistance, and so seems to miss Galileo's point. Chapter 5, however, entitled Observation and Experimentation, actually seeks to stress that 'controlled laboratory experiments on a particular phenomenon may not always be easy to perform'; hence the example of a feather and a ball falling to the ground is a fair choice. Chapter 6 is entitled The Role of Human Faith in Science. The 
author advances that we 'trust the textbooks and journal articles that describe e.g. how many planets there are in our solar system and e.g. that atoms support discrete energy levels and so on.' Actually, I would rather emphasize that school laboratory classes in physics, chemistry and biology are essential for students and pupils. Thus they could directly see in their school laboratory key scientific evidence. These direct experiences would ideally include seeing via the eyepiece of a prism spectrometer the discrete spectra of sodium gas emission and use of their school's telescope to view our solar system planets. So, I would argue that the faith of the public should not come into it, indeed that matters of faith would be a concern of religion not science. The strength of science is that one does not have to take someone else's word for it. Chapter 7 is entitled Approximate and Limited Description of Natural Phenomena. This chapter is rather vague in its mission. It seems to be making the obvious point that mankind has not discovered everything as yet (quote: 'Takeaway message: The description of phenomena is always incomplete.'). Chapter 8 is entitled Hypothesis. This would be a cornerstone chapter. Four pages into it, however, the power of hypothesis making in science and discovery is still not articulated; rather it is stated that 'Hypotheses are not objective data or facts.' No, but I would suggest that they are a marvellous instrument for planning new experiments. Chapters 9, 10 and 11 focus on theory. Chapter 10 is promisingly entitled Competing Theories. This chapter has an interesting start: 'Suppose I come up with two distinct theories for a given set of observations... Which one should we choose?' An instructive example that the author could usefully have described would be the two theories of light: light as waves and as photons. An interesting excursion is made by the author into the role of money, funding and clout, i.e. reputation in modern science. The final sentence takeaway message captures the essence: 'If the theories make different predictions, then experiments should settle the issue.' It would have been good to have an important practical example here, for example, is there climate change or not? Chapter 11 is entitled Can One Theory Be 'Derived' from Another? This I found to be a very sound chapter. A telling example was comparing Newtonian mechanics with Einstein's theory of special relativity. The latter has a vital difference: the speed of light is taken as a constant by Einstein, and what does that lead to in consequence? One result was the abandonment of the need for an ether as a medium for light to propagate through, something that fascinated late 19th century physicists. Chapter 12 is entitled Verifying or Falsifying? And What? Clearly this chapter is going to be classic Karl Popper axiom driven. The chapter starts with a very amusing cartoon of two scientists debating the theory of everything: 'does this achievement put us out of work?' Unfortunately, the author does not develop the notion of falsifiability of a theory being more powerful than attempting verifiability of a theory. Chapter 14 is 'Consensus' in Science? What Is That? This chapter provides the chance for the author to introduce Kuhn's paradigm shift as a concept for marking scientific progress. Di Ventra again makes an interesting link with the need to win funding and the fact that for young scientists to get promotion they are compelled to remain within 'the establishment'. In connection with this, I have argued that since funding success rates are consistently never better than one in three, and often worse, it is vital to keep one's personal research, i.e. at-the-bench skills, up to date. That way one can still tackle unfunded ideas and/or the adventurous ones that are so hot one would not delay them with writing of a grant application. Chapter 15 is a Flow Chart of the Scientific Method. The small-format size of the book makes this figure illegible without a magnifying glass, but with it I think its contents look fine. OUP could have done better with the sizing of this figure. Chapter 16 is entitled The 'What' and 'Why' Questions. This is a good chapter as it describes examples of questions which cannot be addressed by science but scientists may well be asked about. Examples given are 'What is gravity?' and 'Why is there gravity?' This leads on to questions posed by the author about the existence of God or intelligent design, thus defining a boundary between science and religion. A popular science news item in recent years has been the Higgs boson as the God particle, i.e. explaining why matter manifests at all, or as Lederman \& Teresi put it The God Particle: If the Universe Is the Answer What Is the Question?' (Dell Publishing, New York, 1993). This could have been a more telling example and certainly one that is raised with me at my tennis club. Chapter 17 is entitled Scientism: Abusing the Scientific Method. This is a somewhat convoluted chapter. Suffice to say it is making the point that there is a difference between science and religion. Another way of making the point raised, and the one usually adopted by scientists, is that religion involves matters of faith whereas science is objective, which the author has stressed earlier in his book. Chapter 18 is Final Thoughts. This chapter concludes on an excellent note: 'if the scientific method is applied with intellectual honesty, it will allow us to make sense of the facts that Nature will reveal in the process of discovery ... objective reality is there.'

In summary I found this book good in parts. Such a short book, with its clear takeaway messages, is needed. A general question I would pose: is there one scientific method? Certainly the making of a hypothesis is one, stressed by this book. There are others. There is empirical gathering of facts from which a law (in physics) may be deduced. In biology, what about the making of a collection as Darwin did or, in its modern guise, sequencing all the genes of a genome with no initial hypothesis in mind? After the collection is made, progress can be achieved by intellectual reflection. There is also the case where we ask what if we do this or that in our experiments, i.e. following a hunch, not as firm as a hypothesis. Then there is asking a question such as Einstein's 'What if the speed of light is finite?', from which a mathematical theory flows. In a further approach, that involving statistics, CERN, for example, insisted on a ' 5 sigma' level of proof for the Higgs boson. Another feature of this book is the writing style. The back cover states that the book is 'written in a clear, concise, and colloquial style'. Should colloquialisms in fact be avoided in presenting to audiences from around the globe so as to avoid misunderstandings and be clear? That said, each chapter usefully concludes with a list of take home messages. 